\title{
Effects of disorder on the blockade voltage of two-dimensional quantum dot arrays
}

\author{
Heinz-Olaf Müller, ${ }^{\text {a) }}$ Kozo Katayama, and Hiroshi Mizuta \\ Hitachi Cambridge Laboratory, Cavendish Laboratory, Madingley Road, Cambridge CB3 OHE, \\ United Kingdom
}

(Received 26 May 1998; accepted for publication 18 August 1998)

\begin{abstract}
The influence of both geometric and offset charge disorder of two-dimensional quantum dot arrays (also known as network tunnel junctions) on their Coulomb blockade voltage $V_{b}$ is studied using extensive Monte-Carlo simulations. A general increase of $V_{b}$ with increasing disorder is confirmed, but an exception to the rule is found for intermediate degrees of offset charge disorder. Detailed studies of the $V_{b}$ distribution reveal a stability of its minimal value against geometric disorder, whereas this figure is considerably increased for high offset charge disorder. Implications of our results for single electron device design are discussed. (C) 1998 American Institute of Physics.
\end{abstract} [S0021-8979(98)05022-1]

\section{INTRODUCTION}

Nanoscale integrated electronics is still close to the boundary between the possible and the impossible, and is therefore very prone to errors caused by unavoidable side effects. Different kinds of disorder, as studied in this article, are one example.

Disorder is an intrinsic ingredient of nearly all single electron circuits fabricated so far, especially those which rely on natural lithography. ${ }^{1,2}$ Therefore, a fair amount of effort has already been invested into both theoretical and experimental studies of disorder effects in single electron structures. The main objectives of this work are the determination of the blockade voltage $V_{b}$, which corresponds to the maximal bias voltage at which current at zero temperature is totally suppressed due to Coulomb repulsion, and the exponent $\zeta$, which describes the current for bias voltages slightly above $V_{b}, I \propto\left(V-V_{b}\right)^{\zeta}$.

Of the different types of disorder, offset charges have caused by far the most interest. In one-dimensional arrays, the influence of offset charge on $V_{b}$ has been studied both experimentally ${ }^{3,4}$ and theoretically. ${ }^{5}$ The current dependence $I \propto\left(V-V_{b}\right)^{\zeta}$ resulting from the effect of background charges was suggested ${ }^{6}$ using $\zeta=1$ in case of one-dimensional arrays, and $\zeta=5 / 3$ in case of two-dimensional networks. That work has spawned a number of experiments ${ }^{7-11}$ with in part different values of $\zeta$.

The influence of disorder on Coulomb blockade systems has also been studied in terms of the metal-insulator transition. ${ }^{12-14}$ The system considered differs, however, from the classical Coulomb blockade situation in so far as the conductance is much higher. In fact, it is the conductance that triggers the transition rather than an applied bias voltage.

In the present article we investigate the influence of disorder on $V_{b}$ in the case of two-dimensional single electron networks by means of numerical simulation. In Sec. II we describe our model system and the employed realization of

\footnotetext{
${ }^{a)}$ Electronic mail: hom@phy.cam.ac.uk
}

disorder. The following Sec. III gives a detailed overview of our numeric tools and Sec. IV contains the discussion of our results.

\section{THE SYSTEM UNDER CONSIDERATION}

For the purpose of this study, emphasis is put on the general geometric realization of Coulomb blockade devices rather than a particular material or a certain technology. The (regular) basic system of our simulations is shown in Fig. 1. It consists of an $6 \times 4$ array of planar rectangular dot electrodes, each of which is nominally $5 \times 5 \mathrm{~nm}^{2}$ in size and placed on a $7 \mathrm{~nm}$ grid. Three leads are attached to the array: source (left), drain (right), and gate (bottom). Similar computations with circular dots are performed for reference. The choice of the system size is a trade off between what is realized in experiment and what can be handled numerically. The parameters of dot size and dot distance correspond to typical values of both semiconducting and metal dots produced by diverse techniques. The bias is applied to the source electrode only whereas the drain is grounded. This simulates the operation of a memory cell, where the applied word voltage "writes" charge into the memory node.

Two different types of disorder in this system are studied: geometric disorder and offset-charge disorder. Geometric disorder is understood as fluctuations in position and size of the individual dots. In the language of localization ${ }^{15}$ this corresponds to topological and compositional disorder, respectively. Offset charges cause a different kind of compositional disorder, where the site energies are changed by the local field distribution rather than the dot's capacitance.

Position disorder is simulated by changing the dot position from the regular $7 \mathrm{~nm}$ grid. Thus, the maximal cell size within which each dot might move, independent of the other dots, is $7 \times 7 \mathrm{~nm}^{2}$-not so much larger than the dot size itself $\left(5 \times 5 \mathrm{~nm}^{2}\right)$. This matches an experimental situation, where the tunnel resistance is expected to increase exponentially with the interdot distance and thus strongly suppresses the tunnel current. The ratio of the actual cell size to its maxi- 

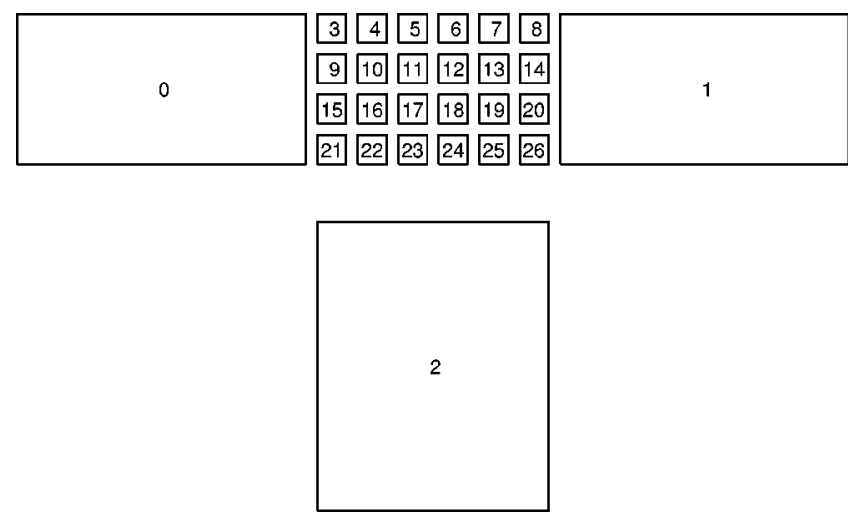

FIG. 1. Regular two-dimensional $6 \times 4$ dot array the disordered derivatives of which are studied in this article. The leads (source, gate, drain-from left) are not affected by disorder.

mum is used as a measure of the degree of position disorder. The position of any dot within its cell is evenly, but randomly distributed. More sophisticated models might be realized easily but the question to what extent they are closer to the real world remains open.

Size disorder is simulated in a very similar way, however the dots' centers are pinned on the $7 \mathrm{~nm}$ grid. Their edge length is varied within a given range, independently from dot to dot and for both dimensions of each dot. The possible range of dot dimensions to its maximum defines the degree of disorder.

Offset-charge disorder is studied by two different approaches. In the first we assign a value of charge directly to each dot in the array whereas in the second the charge on an additional small "impurity" dot influences corresponding charges on the regular dots. According to the $e$ periodicity of the offset-charge behavior the offset charge in the former case is chosen out of the charge interval $[-e / 2: e / 2]$. In contrast the impurity in the latter case can be charged to any value since its charge is excluded from tunneling.

The setup of the offset-charge configuration in the former case starts with an arbitrary choice of the charge on the first dot in the array (No. 3 in Fig. 1). The values for the other dots are chosen in such a way that the offset charge difference between two neighboring dots does not exceed a certain maximum. The ratio of this maximum to the mentioned limit $e$ allows us again to define a degree of disorder. All possible offset-charge values within the predefined range have identical probability of being selected. The purpose of this offset-charge selection scheme is the incorporation of both offset-charge correlation between close dots and independent variation of the common offset-charge level.

\section{EMPLOYED METHOD}

The computation procedure comprises essentially two steps: capacitance calculation and current calculation. First, the geometry of the array under consideration is specified. This includes both kinds of geometric disorder as explained above. Given the geometry of the array and its leads, the complete capacitance matrix is computed which allows not only the determination of the interelectrode cross capaci- tances but also of the stray capacitance of each electrode. In a next step, these stray capacitances are charged by an attached voltage source in order to simulate a certain offsetcharge configuration. Finally, a Monte-Carlo computation reveals the current through the setup as a function of the bias voltage.

The capacitance computation starts from the known capacitance of two planar homogeneously charged rectangular electrodes in a common plane: ${ }^{16}$ the substrate surface. These basic building blocks are assembled to form larger electrodes of different (still planar) geometry and varying charge concentration. Thus, a coarsening procedure is inevitably part of our capacitance program. In order to minimize its influence on the final results and to keep the computational effort within reasonable limits, checks were done resulting in an approximate size of $1.5 \times 1.5 \mathrm{~nm}^{2}$ for our building blocks.

The substrate is assumed to fill the semi-infinite space beneath the common plane of all electrodes, and one dielectric constant is used to describe its influence on the electrostatic field. Similarly, the space above the plane of electrodes is given another constant. Symmetry allows further simplification to one homogeneous medium all around the electrodes of arithmetic-mean dielectric constant. The presented results are calculated for a silicon substrate $\left(\varepsilon_{r} \approx 11.9\right)$ and $\mathrm{SiO}_{2}$ passivation layer $\left(\varepsilon_{r} \approx 3.9\right)$ resulting in a mean value of $\varepsilon_{r}$ $=7.9$.

A difficult point of capacitance computation, but irrelevant to the consideration here, is the difference between measured and computed capacitance values. ${ }^{17,18}$ The presentation of purely simulation results allows us to ignore this problem for the time being.

The cutoff of the macroscopic leads ${ }^{18}$ is another issue to be addressed: since the capacitance of a wire-like lead increases approximately proportionally to its length and the potential inhibits an 1/r drop off, even parts of the leads far away from the central dot array contribute significantly to the potential near the end of the wire. Hence cutoff lengths reach tremendous values in the $\mu \mathrm{m}$ range. ${ }^{18}$ For our much smaller dots, however, we expect correspondingly shorter cutoff lengths. In detail, we extract a ratio between cutoff length and dot size of about 10 from Ref. 18. Correspondingly for our nominally $5 \times 5 \mathrm{~nm}^{2}$ dots an estimated cutoff length 50 $\mathrm{nm}$ is used.

As well as all interelectrode capacitances, the computation of the whole capacitance matrix allows the estimation of the stray capacitances of each electrode. This is an essential ingredient of single-electron electronics simulation as pointed out recently, ${ }^{19}$ since even for electrodes of moderate size the stray capacitance exceeds the apparently ultrasmall tunnel capacitances. The value of the stray capacitance follows from that of the total capacitance minus all cross capacitances of the electrode under consideration.

In Fig. 2 computed self-capacitances are displayed for rectangular dots. Changing the shape of the dot while its area is fixed results in increased capacitance. For increasing dot area a power-law increase of the capacitance is observed (see inset).

The realization of a given offset-charge configuration of the dot array, which is decided beforehand, makes use of the 


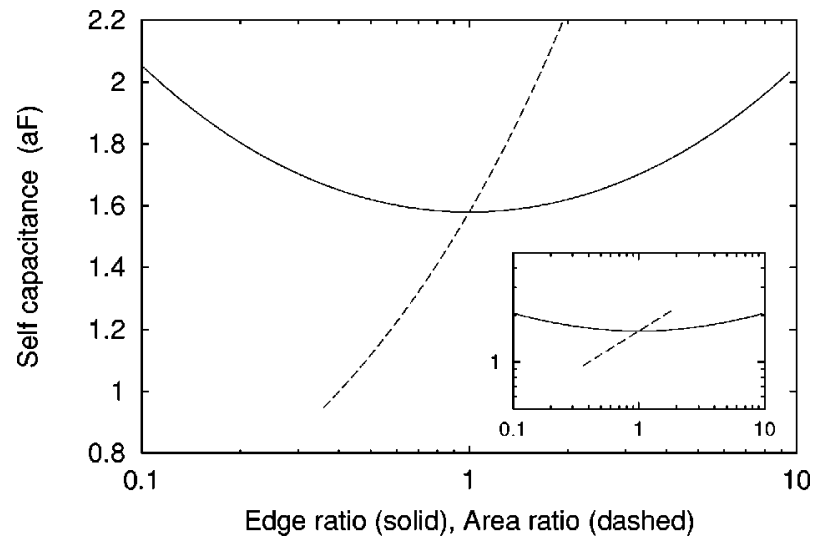

FIG. 2. Self-capacitance of a rectangular dot as function of its edge ratio (solid curve). The dot area is fixed at $25 \mathrm{~nm}^{2}$. The dashed line shows the area dependence of a quadratic dot as function of the dot area. The inset shows the same data in double-log representation.

stray capacitances mentioned above. As depicted schematically in Fig. 3, an individual voltage source is taken to be connected to each dot's stray capacitance. The voltage value is adjusted so that the expected charge value is induced on the dot. No offset charge is considered for the macroscopic leads.

Besides all the capacitances, tunnel resistances are a necessary input for the Monte-Carlo current calculation. However, as long as only the blockade voltage $V_{b}$ is considered, it is independent of the choice of the resistances. ${ }^{5}$ This is because the determination of $V_{b}$ poses an electrostatic problem without flowing current. A constant tunnel resistance of $500 \mathrm{k} \Omega$ is used for each tunnel junction and the independence of the outcome of this value was checked numerically. This value is large in comparison to the resistance quantum $R_{Q}=h / e^{2} \approx 25.79 \mathrm{k} \Omega$ and justifies the use of the so-called orthodox theory of single-electron tunneling. ${ }^{20}$

The Monte-Carlo simulation program is in general similar to other single-electron simulators described extensively in recent literature. ${ }^{21-25}$ The duration of a specific charge state $\Delta t$ is modeled by a stochastic Poisson process, $\Delta t$ $=-\log (r) / \Gamma$, where $r \in[0: 1]$ is a random number and $\Gamma$ is the tunnel rate. This rate in turn follows from the difference of free energy between initial and final state of the system $\Delta F$ which is calculated from the capacitance setup:

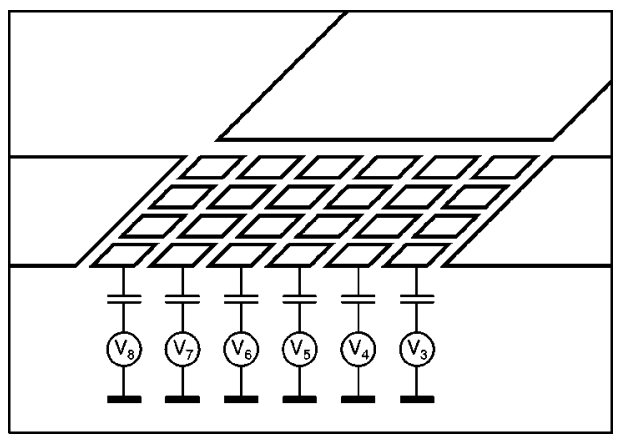

FIG. 3. Realization of a certain offset-charge realization by means of voltage sources charging up the individual stray capacitances of each dot in the array. Only the first line of voltage sources is shown schematically.

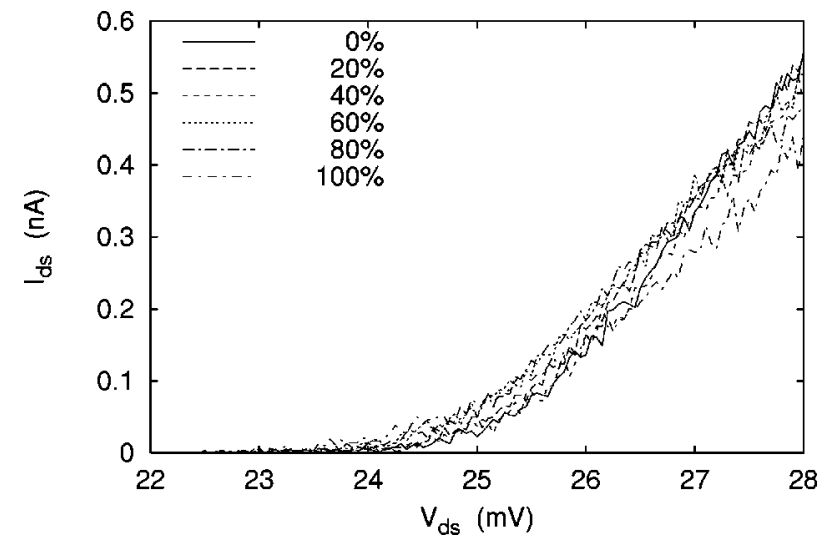

FIG. 4. Source-drain current for different levels of position disorder ranging from $0 \%$ (regular array) to $100 \%$ (maximal disorder).

$$
\begin{aligned}
& \Gamma=\frac{k_{B} T}{e^{2} R} \frac{x}{1-\exp (x)}, \\
& x=\Delta F /\left(k_{B} T\right), \\
& k_{B} \text { Boltzmann's constant, } \\
& T \text { temperature, } \\
& e \quad \text { Electronic charge, and } \\
& R \text { tunnel resistance. }
\end{aligned}
$$

This approach is incapable of describing rare tunnel events adequately since in these cases $\Gamma$ becomes very small and the system gets locked in one state. In our simulation $\Delta t$ is limited for practical reasons to $0.1 \mu$ s resulting in a minimal nonvanishing current of $e / 0.1 \mu \mathrm{s} \approx 1.6 \mathrm{pA}$, where one electron only is transferred during all $\Delta t$. On one hand this procedure might seem rather crude, however, the neglect of higher-order tunnel processes (cotunneling) imposes further deviations in the low-current domain of the simulations. On the other hand the current discretization provides a unique means of extracting the blockade voltage $V_{b}$ from an $I_{d s}-V_{d}$ characteristic: $V_{b}$ is assigned the largest value of $V_{d}$ for which the simulation results in $I_{d s}=0$.

The importance of sufficiently low temperature in single-electron tunneling was pointed out even in the very early articles on this subject. ${ }^{26}$ In order to observe singleelectron effects despite thermal fluctuations $k_{B} T \ll e^{2} /(2 C)$ is required, where $C$ is given by the total capacitance of the dot. In our case, the computed capacitance is $1-2 \mathrm{aF}$ for the $5 \times 5 \mathrm{~nm}^{2}$ dots corresponding to an energy of $40-80 \mathrm{meV}$. In contrast, a temperature of $4.2 \mathrm{~K}$ is used in all our simulations, which corresponds to only $360 \mu \mathrm{eV}$.

\section{RESULTS AND DISCUSSION}

\section{A. Position disorder}

In Fig. 4 we present typical results of the whole numeric procedure described above. In the figure only the voltage range near $V_{b}$ is shown, however tests have run to assure current suppression in the whole blockade range. The fluctuating behavior of the current is attributed to the employed 


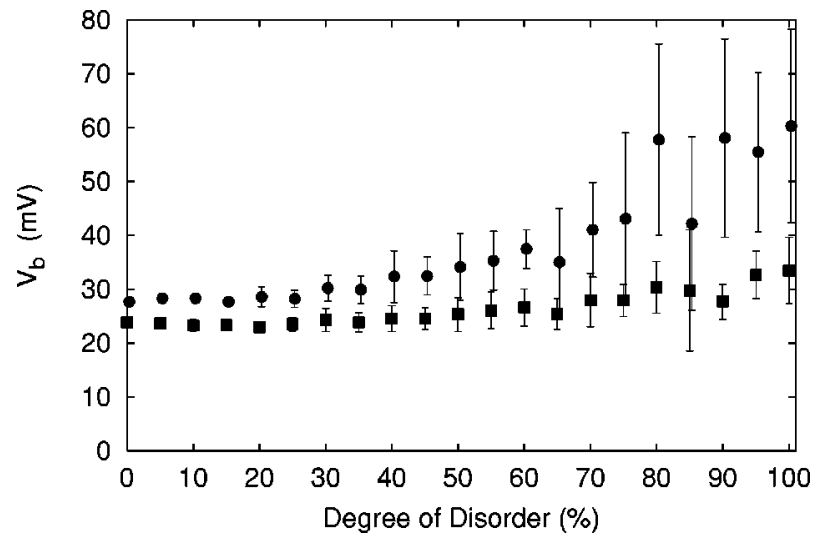

FIG. 5. Blockade voltage $V_{b}$ as function of the degree of size disorder for arrays of rectangular ( $\mathbf{\square})$ and circular (O) dots. A slight horizontal offset is used for clarity.

Monte-Carlo algorithm. The figure shows that the current discretization in steps of $1.6 \mathrm{pA}$ does not hinder the computation and the evaluation of its results.

Different curves in Fig. 4 display different levels of position disorder. According to the outlined method of determination of the blockade voltage only a small decrease of $V_{b}$ from 24 to $23 \mathrm{mV}$ is found for the transition from the regular case to the maximal disordered one. This disorder stability of $V_{b}$ is due to the fact that the actual dot positions cannot deviate very far from their nominal position owing to the exponential dependence of the tunneling resistance. Hence, the variation in capacitances is comparably small and especially the dominating stray capacitances are almost the same for all dots. In this sense, the system is still very much free of disorder and this is represented in the results of Fig. 4.

\section{B. Size disorder}

The results for geometric size disorder are shown in Fig. 5. For each degree of disorder, the $V_{b}$ of ten samples were computed and the symbols indicate the mean value of the blockade voltage. In order to guide the eye error bars are attached to each symbol corresponding to the size of standard deviation. For as few as ten samples, however, the standard deviation is only a fraction of the statistical error. Data for rectangular and circular dots are presented. The higher $V_{b}$ of the latter is understood geometrically: the nominal diameter corresponds to the edge length of the rectangular dots. Thus, smaller area causes smaller capacitance and in turn higher blockade voltage.

With increasing disorder both $V_{b}$ and its standard deviation increases. The suppression of the conductance with increasing disorder is a very general feature of disorder induced localization. ${ }^{15}$ It is studied in terms of the pinning of charge solitons as well. ${ }^{27,4}$ The large error bars near maximal disorder indicate a wide distribution of $V_{b}$ values. We investigated this range in Fig. 6 in more detail. Here we use 100 samples of rectangular dots per degree of disorder which allows a better statistic evaluation. The histogram uses 25 classes. Despite the fluctuation it is seen that for all cases the mean value of the distribution is near its minimal value. The latter is constantly in the range $20-25 \mathrm{mV}$ which is the value

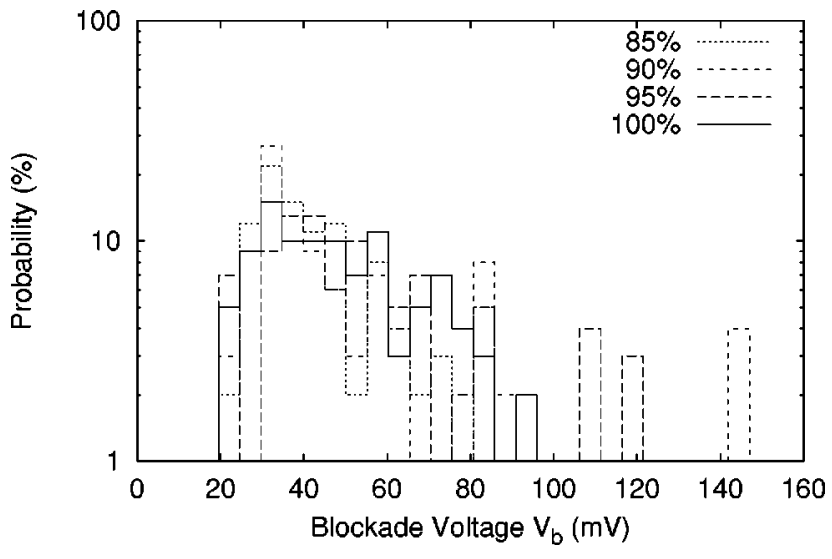

FIG. 6. Distribution of the blockade voltage for four different degrees of high geometric size disorder.

of the regular case. The data strongly suggests that size disorder does not affect the minimal blockade value of twodimensional (2D) Coulomb blockade structures.

These results can be understood in terms of dominating stray capacitances. The regular dot array of uniform dots, Fig. 1, causes the disorder-independent minimal value of $V_{b}$. On the other hand, the maximal value is governed by the smallest dot in the current path and the probability of the existence of small dots increases with increasing disorder. In contrast to this, large dots are of minor influence on $V_{b}$ in case of quantum dot arrays.

With regard to the application of Coulomb blockade devices minimal and maximal values of $V_{b}$ are of interest. In a memory cell, ${ }^{28}$ for instance, minimal $V_{b}$ determines the retention time and the ratio of word voltage and maximal $V_{b}$ the read/write time. Therefore our conclusion is twofold: first, the retention time problem is nearly independent of size disorder; second, in order to achieve reliable read/write times the word voltage should be almost ten times as large as the regular blockade voltage due to large fluctuations of the maximal $V_{b}$ value.

\section{Offset-charge disorder (I)}

In Fig. 7 the blockade voltage for different offset-charge configurations is shown. The results for arrays of round dots

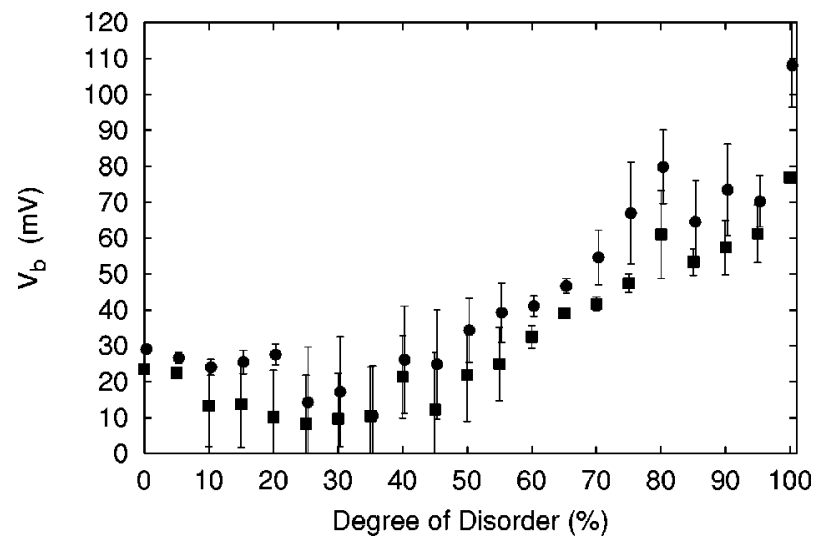

FIG. 7. Blockade voltage $V_{b}$ as function of degree of offset-charge disorder for arrays of rectangular $(\boldsymbol{\square})$ and circular $(\mathbf{O})$ dots, ten samples per point. 


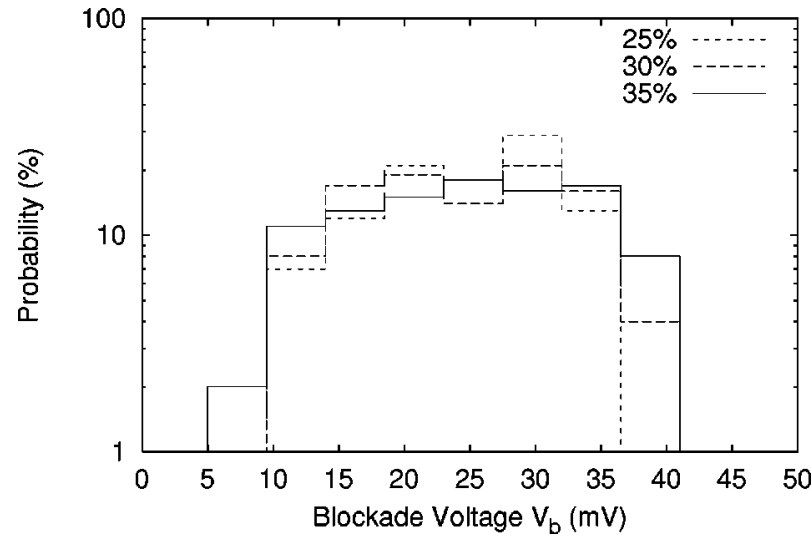

FIG. 8. $V_{b}$ statistics for intermediate degrees of offset-charge disorder of 100 samples $(25 \%-35 \%)$.

are given for comparison again. Starting from the original value of the regular system an initial drop off of $V_{b}$ is followed by a strong increase at high degrees of disorder eventually reaching about three times the original value. Again, the evaluation makes use of only ten samples per degree of disorder which probably does not reveal the whole picture. Two different ranges are therefore studied in detail: the intermediate range of reduced $V_{b}$ and the high-disorder range of enhanced blockade voltage.

Figure 8 shows a histogram of $V_{b}$ in the intermediate disorder range $(25 \%-35 \%)$. The statistics used 100 samples again. An overall reduction of the blockade voltage is obvious. The origin of this reduction is found once more in the effect of large stray capacitances. Since the drain electrode is grounded they cause a drastic capacitance asymmetry resulting in a wide offset-charge range of almost no blockade. This effect is not limited to two-dimensional arrays of dots, but can also be observed in a single dot as shown in Fig. 9.

Furthermore, in a dot array the breakdown region of the blockade voltage around $Q_{0} \approx \pm e / 2$ increases and thus causes smaller values of $V_{b}$. For the array, Fig. 9 shows the blockade voltage with identical offset charge on each dot (lower solid curve). No disorder is included in this calculation. The upper solid curve in displays $V_{b}$ for "flipping" values of $Q_{0}$ in source-drain direction, i.e., from left to right in Fig. 1. The dots of each row carry identical offset charges again. This choice simulates the case of a particularly high disorder and leads-as discussed below-eventually to higher values of $V_{b}$. Here we want to point out that for intermediate degrees of disorder in Fig. 7 small $\left(Q_{0} \approx\right.$ $-0.3 e)$, but still reasonably uniform offset-charge values are possible which cause the observed behavior of Coulomb blockade reduction.

In Fig. 10 the results for large offset-charge disorder are presented. These confirm the first glimpse from Fig. 7: Both minimal and maximal value of $V_{b}$ increase with disorder, but the width of the $V_{b}$ distribution hardly changes. To determine the shape of the distribution, however, significantly more than the studied 100 samples are necessary.

The increase of the blockade voltage for high degrees of disorder can be understood by means of the model system used in Fig. 9 since considerable jumps of the offset charge

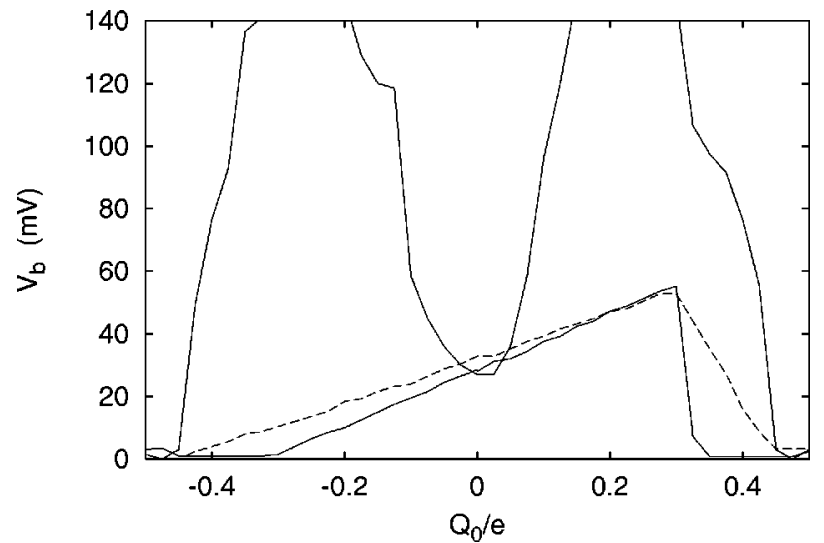

FIG. 9. Maximal and minimal blockade voltage $V_{b}$ as function of the offset charge (solid lines). For the minimal value of $V_{b}$ each dot carries the same value of $Q_{0}$. For maximal $V_{b}$ it is alternating in source-drain direction. The dashed line displays the result for a single dot as reference.

from dot to dot become more and more probable. The situation of Fig. 9, where the offset charge alternates between two values, is from this point of view extreme, but instructive. The different offset charges shift the electrostatic potential of the corresponding dots up and down which allows tunneling electrons to move easily from a high-potential dot to a lowpotential one but significantly suppresses the next tunneling event from low to high potential. Therefore charges get stuck on every second dot and only a considerable increase of the bias voltage will allow them to move on.

We have checked that this behavior is determined by the background charge distribution in the source-drain direction only, by considering arrays of different width. All of them produce very similar results. As expected, the situation is symmetric for $Q_{0} \rightarrow-Q_{0}$ which causes a change of sign for each offset charge and corresponds also to the situation of negative bias. For $Q_{0}=0$ the results of the "clean" array are reproduced, whereas for $Q_{0} \approx \pm e / 2$ a breakdown of the Coulomb blockade is observed. The latter indicates the general $e$ periodicity of offset-charge dependencies.

From Fig. 9 and from the used definition of disorder in Sec. II quantitative limits for the range of suppressed and enhanced Coulomb blockade can be determined. A minimal

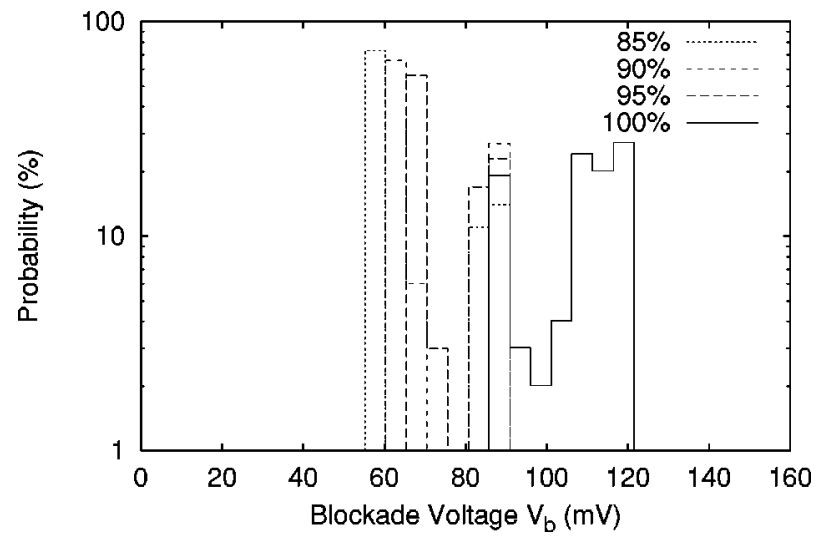

FIG. 10. $V_{b}$ statistics for high degrees of offset-charge disorder. Plotted are the results for 100 samples of rectangular dots. 


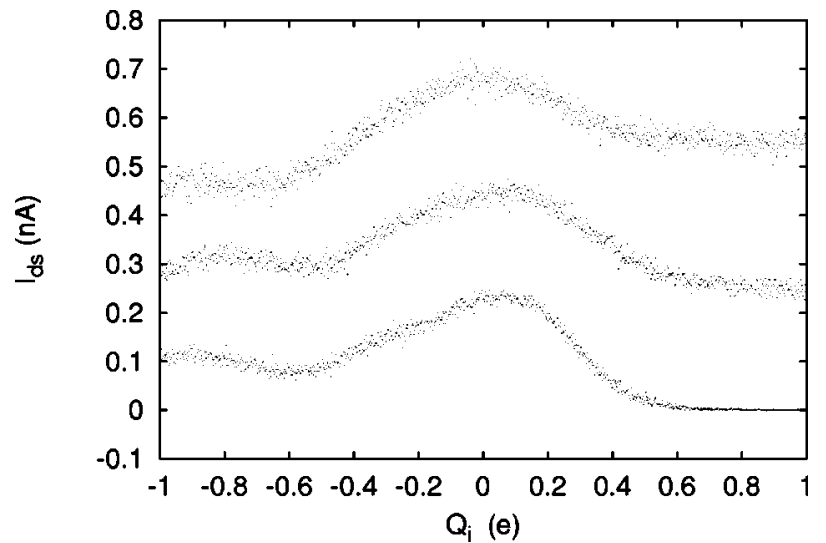

FIG. 11. Current through a regular dot array as a function of the charge $Q_{i}$ on a small additional impurity dot in its center. The array is six dots long and six, four, or two dots wide (from top). The applied bias is about $30 \mathrm{mV}$ which is approximately $5 \mathrm{mV}$ above $V_{b}$ for this type of structure.

disorder value of $20 \%$ is necessary to reach an offset charge of $-0.3 e$ which leads to blockade suppression. In case of a homogeneous charged quantum dot array this suppression domain covers about $40 \%$ of all offset-charge values. Hence, $V_{b}$ is reduced for disorder between about $20 \%$ and $40 \%$ whereas higher degrees of disorder result in increased $V_{b}$ values.

\section{Offset-charge disorder (II)}

Another approach to the study of offset-charge disorder is presented in Fig. 11. As outlined above, the influence of a charged impurity on the current through the setup is investigated. The current for three different arrays of $6 \times 6,6 \times 4$, and $6 \times 2$ dots (from top) as a function of the impurity charge $Q_{i}$ is displayed. The three curves differ mainly by a constant shift in current which is attributed to the additional dots outside the central two rows. This suggests that the impurity charge is effectively screened by its nearest neighbors. The strong screening is caused by large stray capacitances in comparison with the tunnel capacitances. Highly charged impurities suppress the current and might even pinch it off as in the case of the narrow $6 \times 2$ system. As discussed above, this effect can be understood following the line of $V_{b}$ enhancement by offset charges or in terms of localization caused by disorder as well. ${ }^{15}$

\section{CONCLUSION}

In conclusion, we have presented detailed numeric studies of the effect of disorder on the blockade voltage of twodimensional quantum dot arrays. Given a geometric realization (if necessary with offset charges), both capacitances and blockade voltage are computed. We find a dominant influence of the stray capacitances which turned out to be larger than the tunnel capacitances. Therefore, neither position disorder nor size disorder has a significant effect on the minimal value of $V_{b}$. In contrast the mean value increases in case of size disorder whereas position disordered systems behave almost like the regular one.
Offset charges cause a breakdown of the Coulomb blockade in an intermediate range of disorder, but $V_{b}$ increases significantly for high offset-charge disorder. In this case the width of the $V_{b}$ distribution increases slower thus mainly causing a shift of the distribution to larger values with increasing disorder. The intermediate breakdown is shown to be due to the capacitance asymmetry caused by the dots' stray capacitances which allow small blockade voltages for small $(\sim-e / 2)$ but uniform offset-charge values. The blockade enhancement is an effect of large disorder which pins tunneling charges within the dot array.

With regard to their device application, single-electron systems shall have a well-defined Coulomb blockade. In particular, the minimal blockade voltage should have a sharp limit and a reasonably small maximal value is required for competitive performance. For the cases studied here we find $V_{b}$ to be independent of position disorder and its minimal value independent of size disorder. However, care must be taken to avoid certain correlated values of offset charges since they will cause a blockade breakdown. Since in general offset charges cannot be avoided, decorrelation of them might be feasible not only to circumvent the breakdown problem but also to increase the blockade voltage considerably.

\section{ACKNOWLEDGMENTS}

This work was performed within the ESPRIT MEL-ARI project FASEM (Fabrication and Architecture of SingleElectron Memories). We thank David A. Williams for many valuable discussions.

${ }^{1}$ H. W. Deckman and J. H. Dunsmuir, Appl. Phys. Lett. 41, 377 (1982).

${ }^{2}$ C. Haginoya, M. Ishibashi, and K. Koike, Appl. Phys. Lett. 71, 2934 (1997).

${ }^{3}$ P. Delsing, T. Claeson, K. K. Likharev, and L. S. Kuzmin, Phys. Rev. B 42, 7439 (1990)

${ }^{4}$ P. Delsing, in Single Charge Tunneling: Coulomb Blockade Phenomena in Nanostructures, NATO ASI Series B: Physics, Vol. 294, edited by H. Grabert and M. H. Devoret (Plenum, New York, 1992), pp. 249-274.

${ }^{5}$ J. A. Melsen, U. Hanke, H.-O. Müller, and K. A. Chao, Phys. Rev. B 55, 10638 (1997), cond-mat/9611052.

${ }^{6}$ A. A. Middleton and N. S. Wingreen, Phys. Rev. Lett. 71, 3198 (1993).

${ }^{7}$ A. J. Rimberg, T. R. Ho, and J. Clarke, Phys. Rev. Lett. 74, 4714 (1995).

${ }^{8}$ F. Ladieu, M. Sanquer, and J. P. Bouchaud, Phys. Rev. B 53, 973 (1996).

${ }^{9}$ L. Clarke, M. N. Wybourne, M. Yand, S. X. Cai, and J. F. W. Keana, Appl. Phys. Lett. 71, 617 (1997).

${ }^{10}$ M. N. Wybourne, L. Clarke, M. Yan, S. X. Cai, L. O. Brown, J. Hutchison, and J. F. W. Keana, Jpn. J. Appl. Phys., Part 1 36, 7796 (1997).

${ }^{11}$ P. Delsing, C. D. Chen, D. B. Haviland, T. Bergsten, and T. Claeson, cond-mat/9805121 (unpublished).

${ }^{12}$ C. A. Stafford and S. D. Sarma, Phys. Rev. Lett. 72, 3590 (1994).

${ }^{13}$ C. I. Duruöz, R. M. Clarke, C. M. Marcus, and J. S. Harris, Phys. Rev. Lett. 74, 3237 (1995).

${ }^{14}$ H. Chen, J. Wu, Z.-Q. Li, and Y. Kawazoe, Phys. Rev. B 55, 1578 (1997).

${ }^{15}$ B. Kramer and A. MacKinnon, Rep. Prog. Phys. 56, 1469 (1993).

${ }^{16}$ H. Pothier, Ph.D. thesis, University of Paris 6, 1991, 3.2.1 Calculation of Planar Capacitances (unpublished), p. 46.

${ }^{17}$ K. A. Matsumoto, K. K. Likharev, P. Dresselhaus, L. Ji, S. Han, and J. Lukens, J. Appl. Phys. 81, 2269 (1997); cond-mat/9609273.

${ }^{18}$ M. Knoll, H. F. Uhlmann, M. Götz, and W. Krech, IEEE Trans. Appl. Supercond. 7, 3524 (1997).

${ }^{19}$ J. G. Lu, J. M. Hergenrother, and M. Tinkham, Phys. Rev. B 57, 4591 (1998).

${ }^{20}$ D. V. Averin and K. K. Likharev, in Mesoscopic Phenomena in Solids, Modern Problems in Condensed Matter Sciences (30), edited by B. L. 
Altshuler, P. A. Lee, and R. A. Webb (Elsevier, Amsterdam, 1991), pp. 173-271.

${ }^{21}$ L. R. C. Fonseca, A. N. Korotkov, K. K. Likharev, and A. A. Odintsov, J. Appl. Phys. 78, 3238 (1995).

${ }^{22}$ K. P. Hirvi, M. A. Paalanen, and J. P. Pekola, J. Appl. Phys. 80, 256 (1996).

${ }^{23}$ R. H. Chen, A. N. Korotkov, and K. K. Likharev, Appl. Phys. Lett. 68, 1954 (1996).
${ }^{24}$ C. Wasshuber and H. Kosina, Superlattices Microstruct. 21, 37 (1997).

${ }^{25}$ S. Amakawa, H. Majima, H. Fukui, M. Fujshima, and K. Hoh, IEICE Trans. Electron. E81-C, 21 (1998).

${ }^{26}$ C. J. Gorter, Physica (Amsterdam) 17, 777 (1951).

${ }^{27}$ N. S. Bakhvalov, G. S. Kazacha, K. K. Likharev, and S. I. Serdyukova, Physica B 173, 319 (1991), preprint JINR-E11-90-177.

${ }^{28}$ K. Nakazato, R. J. Blaikie, and H. Ahmed, J. Appl. Phys. 75, 5123 (1994). 